\title{
Characterization of Biodegradable Polymers with Capacitive Field-effect Sensors
}

\author{
S. Schusser ${ }^{1,2}$, A. Poghossian ${ }^{1,2}$, M. Leinhos ${ }^{1,2}$, P. Wagner ${ }^{3}$, M.J. Schöning ${ }^{1,2}$ \\ ${ }^{1}$ Institute of Nano- and Biotechnologies (INB), FH Aachen, 52428 Jülich, Germany, \\ schusser@fh-aachen.de \\ ${ }^{2}$ Peter Grünberg Institute (PGI-8), Research Centre Jülich GmbH, 52525 Jülich, Germany \\ ${ }^{3}$ Institute for Materials Research, Hasselt University, 3590 Diepenbeek, Belgium
}

\begin{abstract}
A field-effect capacitive sensor has been applied for the real-time in-situ monitoring of degradation of biopolymers for the first time. The sensor is, in principle, capable to detect any changes in bulk, surface and interface properties of polymers induced by degradation processes. The feasibility of this approach has been experimentally proven by using the commercially available biomedical polymer poly $(\mathrm{D}, \mathrm{L}$-lactic acid $)$ as a model system. The degradation of the poly(D,L-lactic acid) was accelerated by changing the degradation medium from neutral $(\mathrm{pH} 7.2)$ to alkaline $(\mathrm{pH} 9)$ condition, resulting in drastic changes in the capacitance of a polymer-modified field-effect sensor.
\end{abstract}

Key words: Field-effect sensor, real-time monitoring, (bio)degradation, poly(D,L-lactic acid)

\section{Introduction}

Although biomaterials have already made an enormous impact in biomedical research and clinical practice (e.g., in drug-delivery systems, as scaffolds for tissue engineering, or orthopedic implants), there is a need for new biodegradable synthetic polymers and implantable devices with controlled/predicted biodegradability [1-5]. For any application, the in-vitro study of the degradation kinetics of the biopolymers is essential not only for a fundamental understanding of the nature of the degradation process but also for the design and optimization of implantable biomedical devices. Common techniques used to quantify degradation (e.g., determination of mass loss, shortening of the chain length or shift of the glass transition temperature towards lower values) are not suitable for real-time measurements because of their destructive manner and thus, limit studies on degradation kinetics in respect of throughput and precision [4]. Analyzing techniques capable for a realtime in-situ monitoring of the degradation kinetics are therefore highly appreciated. In this report, we present experimental results on polymer degradation investigation by means of capacitive field-effect electrolyte-insulatorsemiconductor (EIS) sensors, as a novel and very promising platform for in-vitro testing of biodegradability of polymers. The commercially available biodegradable polymer poly(D,L-lactic acid) (PLA) was used as a model system. The benchmark biopolymer PLA (RESOMER尺)
R 202 H, Evonik Röhm GmbH, Germany) was chosen, because of its exceptional importance, biocompatibility and its use in numerous approved medical devices; and pharmaceutical applications [6]. In previous experiments, fieldeffect sensors have been widely utilized for the detection of various (bio-)chemical quantities, like $\mathrm{pH}$, ion- and analyte concentration, charged macromolecules as well as cellular signals (see e.g., [7-10]).

\section{Sensor principle}

Fig. 1 shows a schematic of the layer structure (a) and a simplified equivalent circuit (b) of the polymer-modified EIS sensor (further referred to as PMEIS) consisting of an Al-Si-SiO2-Ta2O5 structure.

a)

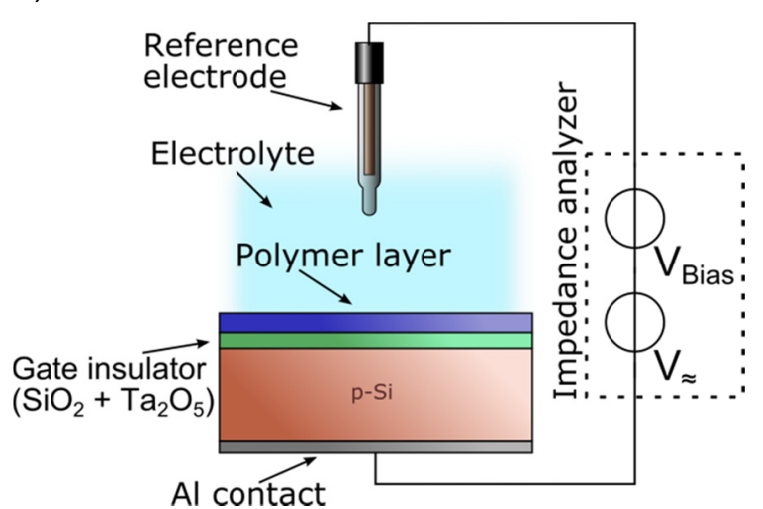


b)

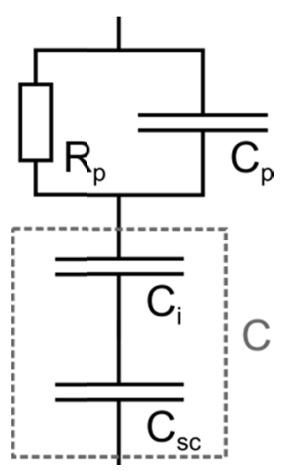

Fig. 1. Schematic of the layer structure (a) and simplified equivalent circuit (b) of a field-effect PMEIS sensor.

For the PMEIS structure, the polymer layer can be described as a parallel network of the polymer geometric resistance, $R_{p}$, and the capacitance, $C_{p}$, which is in series with the bare insulator-semiconductor structure $[10,11]$. The experimentally measured capacitance of the PMEIS structure, $C_{\text {meas }}$, can be expressed as:

$$
C_{\text {meas }}=C \frac{1+R_{p}^{2} C_{p}^{2} \omega^{2}}{1+R_{p}^{2}\left(C C_{p}+C_{p}^{2}\right) \omega^{2}}
$$

where $C$ is the capacitance of the original EIS structure without polymer layer, $C_{i}$ is the gateinsulator capacitance, $C_{s c}$ is the space-charge capacitance of the $\mathrm{Si}, \omega=2 \pi f$ and $f$ is the measuring frequency. Thus, any changes in the polymer resistance/capacitance induced by the polymer degradation will alter the global capacitance/impedance of the PMEIS structure that can be used as an indicator of the polymer degradation.

\section{Fabrication of EIS Sensor and Polymer Deposition}

For the degradation experiments, the EIS chips (with chip size of $10 \mathrm{~mm} \times 10 \mathrm{~mm}$ ) consisting of an $\mathrm{Al}-\mathrm{Si}-\mathrm{SiO}_{2}-\mathrm{Ta}_{2} \mathrm{O}_{5}$ structure $(\mathrm{p}-\mathrm{Si}, \quad \rho=5-$ $10 \Omega \mathrm{cm}, 300 \mathrm{~nm}$ Al as rear-side contact layer, $30 \mathrm{~nm} \mathrm{SiO}{ }_{2} ; 60 \mathrm{~nm} \mathrm{Ta} \mathrm{O}_{5}$ ) were fabricated. The degradable polymer films with different thicknesses were deposited on the $\mathrm{Ta}_{2} \mathrm{O}_{5}$ surface from the polymer solution by means of spin-coating method. The polymer solution was prepared by dissolving the PLA ( $M w=10,000-$ 18,000 ) in acetone. The desired range of layer thickness has been achieved by varying the concentration of the polymer solution (see Fig. 2). The thickness of the polymer layer was defined from profilometry measurements. The deposited polymer films were homogeneous without any visible pores. As an example, Fig. 3 depicts a photo of the PMEIS sensor surface with a $500 \mathrm{~nm}$ thick PLA layer.

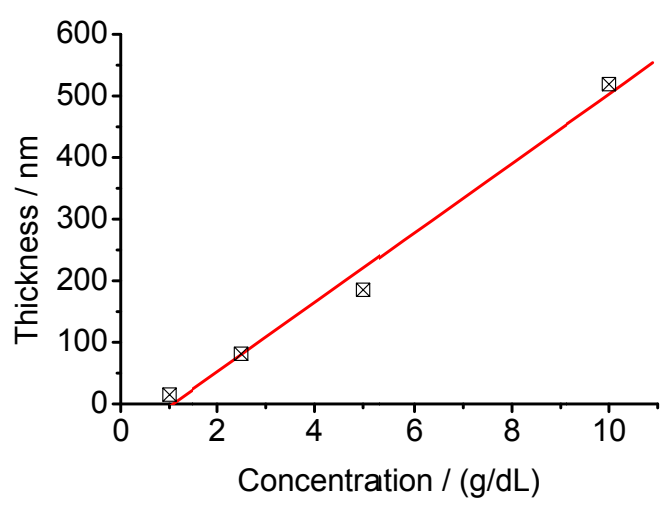

Fig. 2. Thickness of the deposited polymer films as a function of polymer concentration.

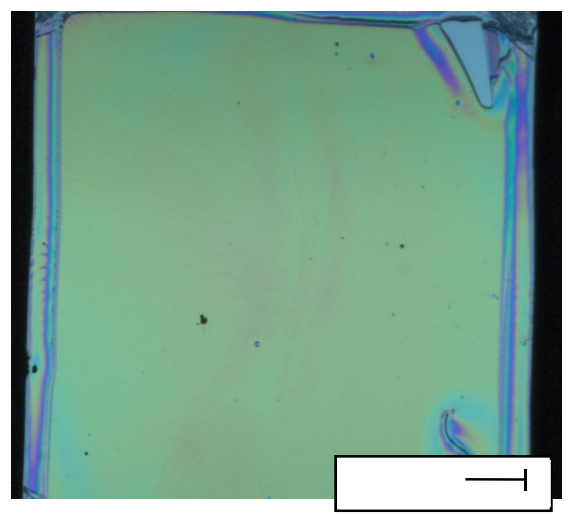

Fig. 3. Photo of the PMEIS sensor surface with a PLA layer of $500 \mathrm{~nm}$ thickness.

The sensor chip was mounted into a homemade measuring cell and then the PMEIS structure was characterized before and during polymer degradation by means of capacitancevoltage $(C-V)$ method. For measurements, a DC polarization voltage is applied between a conventional liquid-junction $\mathrm{Ag} / \mathrm{AgCl}$ reference electrode (Metrohm) and the rear-side $\mathrm{Al}$ contact. A small AC voltage $(20 \mathrm{mV})$ is applied to the system in order to measure the capacitance of the PMEIS sensor. The $C-V$ curves were captured every $1 \mathrm{~h}$ at a frequency of $100 \mathrm{~Hz}$. The degradation experiments were carried out at a temperature of $37^{\circ} \mathrm{C}$.

\section{Results and Discussion}

Fig. 4 represents an example of real-time monitoring of polymer degradation with a fieldeffect structure. In this experiment, the timedependent capacitance of the PMEIS sensor with a $500 \mathrm{~nm}$ thick PLA layer was recorded in the accumulation region of the C-V curve over the time period of about eight days. It is known, that PLA has a slow degradability in neutral $\mathrm{pH}$ solutions and shows a higher degradability in basic solutions [5]. Therefore, during the first 50 $\mathrm{h}$ of monitoring, the polymer layer was exposed to neutral $\mathrm{pH}$ buffer solution of $\mathrm{pH} 7.2$, which 
mimics the physiological conditions of biological fluids. Practically, no changes in capacitance have been observed. Then, in order to accelerate the polymer degradation, the PLA layer was exposed to alkaline buffer solution of $\mathrm{pH}$ 9, resulting in drastic changes in the capacitance of the PMEIS structure over time. The degradation rate can be evaluated from the time-dependent $\mathrm{C}-\mathrm{V}$ curves. These results are supported by optical microscopy of the sensor surface after PLA degradation (Fig. 5).

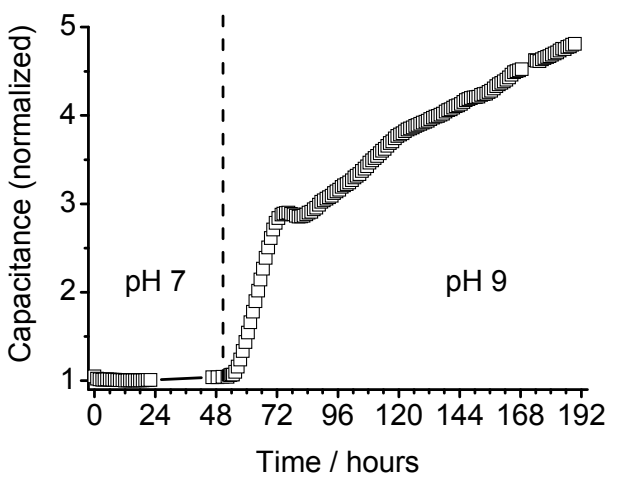

Fig. 4: Monitoring of PLA degradation with capacitive field-effect sensor: time-dependent capacitance changes induced by polymer degradation.

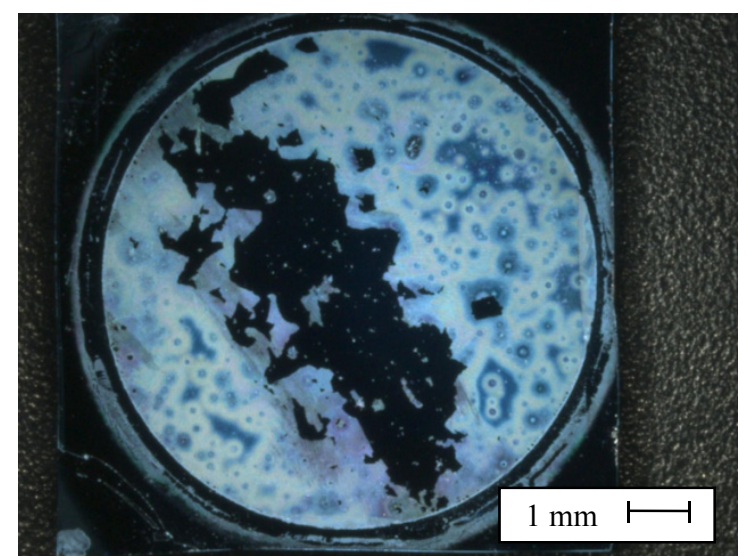

Fig. 5. Optical microscopy image of the PLAcovered sensor surface after polymer degradation.

\section{Conclusions}

The degradation kinetics of the poly(lactic acid) biopolymer was studied using a field-effect capacitive EIS sensor. These preliminary experiments demonstrate the potential of fieldeffect devices as a novel and very promising tool for the real-time in-situ monitoring of polymer degradation. Further works will be directed to optimize the design of the EIS sensor and measuring conditions.

\section{Acknowledgements}

The authors thank the European Union (ERDF) for co-financing this project (BioMiMedics).

\section{References}

[1] L.S. Nair, C.T. Laurencin, Biodegradable polymers as biomaterials, Progress in Polymer Science 32, 762-798 (2007); doi: 10.1016/j.progpolymsci.2007.05.017

[2] M. Martina, D.W. Hutmacher, Biodegradable polymers applied in tissue engineering research: a review, Polymer International 56, 145-157 (2007); doi: 10.1002/pi.2108

[3] [C.-S. Ha, J.A. Gardella, Jr., Surface chemistry of biodegradable polymers for drug delivery systems, Chemical Reviews 105, 4205-4232 (2005); doi: 10.1021/cr040419y

[4] W. Amass, A. Amass, B. Tighe, A review of biodegradable polymers: uses, current developments in the synthesis and characterization of biodegradable polyesters, blends of biodegradable polymers and recent advances in biodegradation studies, Polymer International 47, 89-144 (1998); doi: 10.1002/(SICl)1097-0126(1998100)47:2<89::AID$\mathrm{PI} 86>3.0 . \mathrm{CO} ; 2-\mathrm{F}$

[5] F. von Burkersroda, L. Schedl, A. Göpferich, Why degradable polymers undergo surface erosion or bulk erosion, Biomaterials 23, 4221-4231 (2002); doi: 10.1016/S0142-9612(02)00170-9

[6] K.A. Athanasiou, G.G. Niederauer, C. Agrawal, Sterilization, toxicity, biocompatibility and clinical applications of polylactic acid/ polyglycolic acid copolymers, Biomaterials 17, 93-102 (1996); doi: 10.1016/0142-9612(96)85754-1

[7] M.J. Schöning, A. Poghossian, BioFEDs (fieldeffect devices): State-of-the-art and new directions, Electroanalysis 18, 1893-1900 (2006); doi: 10.1002/elan.200603609

[8] J.R. Siqueira Jr., M.H. Abouzar, A. Poghossian, V. Zucolotto, O.N. Oliveira Jr., M.J. Schöning, Penicillin sensor based on a capacitive fieldeffect structure functionalized with a dendrimer/carbon nanotube multilayer, Biosensors and Bioelectronics 25, 497-501 (2009); doi: 10.1016/j.bios.2009.07.007

[9] A. Poghossian, S. Ingebrandt, A. Offenhäusser, M.J. Schöning, Field-effect devices for detecting cellular signals, Seminars in Cell and Developmental Biology 20, 41-48 (2009); doi: 10.1016/j.semcdb.2009.01.014

[10] Yu. Mourzina, Th. Mai, A. Poghossian, Yu. Ermolenko, T. Yoshinobu, Yu. Vlasov, H. Iwasaki, M.J. Schöning, K+-selective field-effect sensors as transducers for bioelectronic applications, Electrochimica Acta 48, 3333-3339 (2003); doi: 10.1016/S0013-4686(03)00402-X

[11] A. Poghossian, D.-T. Mai, Yu. Mourzina, M.J. Schöning, Impedance effect of an ion-sensitive mambrane : characterisation of an EMIS sensor by impedance spectroscopy, capacitance-voltage and constant-capacitance method, Sensors and Actuators B 103, 423-428 (2004); doi: 10.1016/j.snb.2004.04.071 